Daniele Prado Tormas ${ }^{1}$ Enfermeira, Residência Multiprofissional em Saúde da Família pela Universidade Federal do Mato Grosso (PRENSAF/UFMT) (2016). Rondonópolis/MT, Brasil. E-mail: dany_45@hotmail.com

Débora Aparecida da Silva Santos ${ }^{1}$

Doutora, Curso de Enfermagem, Universidade Federal de Mato Grosso (UFMT), Campus Universitário de Rondonópolis (CUR). Rondonópolis, Mato Grosso. E-mail: deboraassantos@hotmail.com

Gabriela Neves Paula de Souza ${ }^{1}$

Psicóloga, Residência

Multiprofissional em Saúde da Família (PREMSAF).

Universidade Federal do Mato Grosso (UFMT). Rondonópolis, Mato Grosso, Brasil. E-mail: gabrielapsicoarte@gmail.com.

\section{Aliny Fernanda Silva} Canuto Freitas ${ }^{1}$

Farmacêutica, Residência Multiprofissional em Saúde da Família pela Universidade Federal do Mato Grosso (PRENSAF/UFMT) (2016). Rondonópolis/MT, Brasil. E-mail: nandakann@hotmail.com.

\section{Franciane Rocha de} Faria $^{1}$

Doutora, Curso de Medicina, Universidade Federal de Mato Grosso (UFMT), Campus Universitário de Rondonópolis (CUR). Rondonópolis, Mato Grosso, Brasil. E-mail:

francianerdefaria@hotmail.com.

\section{Letícia Silveira Goulart ${ }^{1}$} Doutora, Curso de Enfermagem, Universidade Federal de Mato Grosso (UFMT), Campus Universitário de Rondonópolis (CUR). Rondonópolis, Mato Grosso. E-mail:

Igoulart77@yahoo.com.br.

\section{HIPERTENSÃO E/OU DIABETES MELLITUS EM UMA ESTRATÉGIA SAÚDE DA FAMÍLIA: perfil e associação aos fatores de risco}

\author{
HYPERTENSION AND / OR DIABETES MELLITUS IN A \\ FAMILY HEALTH STRATEGY: profile and association with risk \\ factors
}

\section{RESUMO}

Objetivo: Analisar o perfil epidemiológico e fatores de risco associados de hipertensos e/ou diabéticos de uma unidade de Estratégia de Saúde Família. Método: Estudo transversal, descritivo, de abordagem quantitativa, com 110 hipertensos e/ou diabéticos em Rondonópolis (MT). Foram avaliadas as variáveis sociodemográficas, clínicas, fatores de risco, antropométricas e morbidade auto referida. Foram realizadas análises descritivas dos dados e razão de prevalência. Resultados: A prevalência de HAS e DM foi sexo feminino (58,18\%), faixa etária de 40-59 anos (45,46\%), com companheiro $(67,27 \%)$, baixas renda $(99,19 \%)$ e escolaridade $(75,45 \%)$. Os fatores de risco predominantes foram ingestão de sal 30\% (IC95\%: 0,43-1,63), álcool 6,66\% (IC95\%: 0,09-1,43) e açúcar 10\% (IC95\%: 0,13-1,21). Conclusão: Comparativamente aos homens, as mulheres representam maior prevalência de HAS e/ou DM, destacando baixos níveis de escolaridade e classe social. Quanto aos fatores de risco, demonstrou associação para HAS entre ingestão de sal e álcool e para DM de ingestão de açúcar.

PALAVRAS-CHAVE: Perfil de Saúde; Hipertensão; Diabetes Mellitus; Fatores de Risco; Estratégia Saúde da Família.

\section{ABSTRACT}

Objective: To analyze the epidemiological profile and associated risk factors of hypertensive and / or diabetic individuals from a Family Health Strategy unit. Method: A cross-sectional, descriptive, quantitative study with 110 hypertensive and / or diabetic patients in Rondonópolis (MT). Sociodemographic, clinical, risk factors, anthropometric variables and self-reported morbidity were evaluated. Descriptive analyzes of the data and prevalence ratio were performed. RESULTS: The prevalence of SAH and DM was female (58.18\%), age group of 40-59 years (45.46\%), partner $(67.27 \%)$, low income $(99.19 \%)$ and schooling $(75.45 \%)$. The predominant risk factors were salt intake $30 \%(95 \% \mathrm{Cl}: 0.43-$ 1.63), alcohol $6.66 \%(95 \% \mathrm{Cl}: 0.09-1.43)$ and sugar $10 \%(95 \% \mathrm{Cl}: 0.13-1.21)$. Conclusion: Compared to men, women represent a higher prevalence of $\mathrm{SAH}$ 
and / or DM, highlighting low levels of schooling and social class. Regarding the risk factors, he showed association for SAH between salt and alcohol intake and for DM of sugar intake.

KEYWORDS: Health Profile; Hypertension; Diabetes Mellitus; Risk Factors; Family Health Strategy.

\section{INTRODUÇÃO}

O Brasil atravessa um período de mudança epidemiológica, com profunda transformação no comportamento relacionado ao processo de saúde e doença, que interagem com fatores sociodemográficos, econômicos, ambientais e culturais. Estas alterações na morbimortalidade resultaram em um perfil com predominância de doenças infecciosas e parasitárias para um cenário de prevalência das doenças crônicas não transmissíveis (DCNT). Em 2010, as DCNT resultaram em $73,9 \%$ dos óbitos no país, dos quais $80,1 \%$ tiveram como causa doença cardiovascular, câncer, doença respiratória crônica ou diabetes mellitus. Estima-se que, em 2030, 3/4 de todas as mortes do mundo estarão relacionadas a estas doenças (BRASIL, 2012a; IBGE,2014; CRUZ et al, 2017). As DCNT representam intensa carga de morbidades relacionadas, além de serem responsáveis por alto número de internações e estarem entre as principais causas de amputações, perdas de mobilidade e de outras funções neurológicas. Além disso, caracterizam-se como um problema de saúde pública, pois a cronicidade, a gravidade das complicações e os elementos necessários para controlá-las, tornam estas doenças muito onerosas não apenas para 0 sistema de saúde, como também para os indivíduos afetados e suas famílias. Além disso, os custos intangíveis (dor, ansiedade e perda de qualidade de vida) são difíceis de quantificar e apresentam grande impacto na vida da população acometida (BRASIL, 2013; IBGE, 2014; HOSEY et al, 2014).

Dentre a classificação de DCNT, encontram-se: as doenças cardiovasculares (DCV) como Hipertensão Arterial Sistêmica (HAS), Diabetes Mellitus (DM), câncer e as doenças respiratórias crônicas. A HAS atualmente atinge 32,5\% dos indivíduos adultos, mais de $60 \%$ dos idosos, e até 2025 , deverá aumentar em $80 \%$ nos países em desenvolvimento, como o Brasil. Esta doença também contribui direta ou indiretamente para 50\% das mortes por DCV. Em 2013 ocorreram 1.138.670 óbitos, sendo 339.672 (29,8\%) decorrentes de DCV, considerada como a principal causa de morte no país (SOCIEDADE BRASILEIRA DE CARDIOLOGIA, 2016; SCALA et al, 2015; BRASIL, 2011a).

Além disso, uma epidemia de DM está em curso. Estima-se que a população mundial com esta doença seja da ordem de 415 milhões e que alcance 642 milhões em 2040. Cerca de $80 \%$ desses indivíduos vivem em países em desenvolvimento, com uma maior intensidade e crescente proporção de pessoas acometidas em grupos etários mais jovens. Em 2015, estimou-se prevalência da DM no Brasil em mais de 12 milhões de indivíduos (SOCIEDADE BRASILEIRA DE DIABETES, 2016; INTERNATIONAL DIABETES FEDERATION, 2015).

$\mathrm{Na}$ região Centro-Oeste a HAS foi classificada em terceiro lugar, com maior prevalência de HAS autorreferida (21,2\%) (BRASIL, 2014). Enquanto no Mato Grosso, a prevalência da HAS em pessoas acima de 18 anos é de $22,7 \%$ e do DM 5,6\% (BRASIL, 2012b). Já em Cuiabá, segundo dados do VIGITEL de 2016, observa-se prevalência de HAS de 24,9\% e da DM 7,9\% (BRASIL, 2017). 
A HAS e o DM são importantes fatores de risco para a morbimortalidade cardiovascular. Dados norte-americanos de 2015 revelaram que a HAS estava presente em $69 \%$ dos usuários com primeiro episódio de Infarto Agudo do Miocárdio (IAM), 77\% em Acidente Vascular Encefálico (AVE), 75\% com Insuficiência Cardíaca (IC) e 60\% com Doença Arterial Periférica (DAP). No Brasil, a HAS ainda é responsável por $45 \%$ das mortes cardíacas e $51 \%$ das mortes decorrentes de AVE. Frequentemente, as declarações de óbito não mencionam o DM, pelo fato de que suas complicações, particularmente as cardiovasculares e cerebrovasculares, constituem-se as causas por este tipo de morte (BRASIL, 2013; SOCIEDADE BRASILEIRA DE DIABETES, 2016).

A associação da HAS e do DM é da ordem de $50 \%$, o que não raro, demanda o cuidado das duas doenças no mesmo indivíduo, potencializado pelo fato de que sua concomitância eleva o dano micro e macrovascular, acarretando alta morbidade cardiocerebrovascular. Aliadas, suas complicações (cardíacas, renais e encefálicas) têm alto impacto na perda da produtividade do trabalho e da renda familiar, estimada em US\$ 4,18 bilhões entre 2006 e 2015 (SOCIEDADE BRASILEIRA DE DIABETES, 2016; LIM et al, 2012).

A prevalência destas doenças vem aumentando ao longo dos anos. Segundo dados da Organização Mundial da Saúde (OMS) este fato pode ser atribuído ao crescimento da população mundial, ao envelhecimento populacional, ao processo de urbanização, além da exposição a comportamentos de risco, como maus hábitos alimentares, consumo de álcool, tabaco e exposição crônica ao estresse (WHO, 2013a; SOCIEDADE BRASILEIRA DE DIABETES, 2016; LOBO et al, 2017).

Estudos têm analisado que entre os fatores de risco mais importantes para o aumento da carga de DCNT estão as mudanças nos padrões de comportamento da população, dentre os quais se destacam a inatividade física, o excesso de peso, o consumo abusivo de álcool e o tabagismo, demonstrando a importância da temática como primordial para a criação de estratégias de enfrentamento à problemática. Essas modificações têm-se constituído em desafios aos gestores de saúde em âmbitos local, regional e nacional (PORTELA et al, 2016; GOIS et al, 2016; CRUZ et al, 2017; WHO, 2013b).

Considerando que o correto manejo destas doenças reduz significativamente a morbimortalidade das DCNT, torna-se imprescindível conhecer as características e os fatores de risco associados dos usuários que frequentam os serviços de saúde, bem como a identificação de grupos vulneráveis e prioritários para a elaboração de políticas públicas, a fim de traçar estratégias de ações que melhorem o atendimento a esta população. Tais estudos são fundamentais para o monitoramento das condições de saúde, pois as complicações destas doenças podem ser evitadas por meio da atenção primária à saúde de qualidade (EVANGELISTA, 2013).

Neste contexto, este estudo teve como objetivo analisar o perfil epidemiológico e os fatores de risco associados à hipertensos e/ou diabéticos cadastrados em uma unidade de Estratégia de Saúde Família do município de Rondonópolis-MT.

\section{MATERIAL E MÉTODOS}

Trata-se de um estudo transversal, descritivo, de abordagem quantitativa, realizado em uma unidade de Estratégia de Saúde da Família (ESF) do município de Rondonópolis-MT, no período de dezembro 2016 a junho de 2017. 
Atualmente o município tem cadastrado 38 unidades de ESF, que estão divididas estruturalmente em 05 distritos. O local de coleta de dados foi a ESF Parque São Jorge, localizada no Distrito Sul que atende aproximadamente 3.500 usuários, segundo os dados do Cadastro Nacional de Estabelecimentos de Saúde (CNES). A unidade foi inaugurada no ano de 2007 e possui 1.408 famílias cadastradas, distribuídas em 06 micro áreas. A escolha do local de estudo justifica-se por ser campo de atuação dos profissionais do Programa de Residência Multiprofissional em Saúde da Família (PREMSAF), da Universidade Federal de Mato Grosso (UFMT), Campus Universitário de Rondonópolis (CUR). Primeiramente foi realizado o levantamento prévio dos usuários hipertensos e/ou diabéticos, por meio de dados secundários com análise dos prontuários na unidade de saúde. Foram incluídos no estudo indivíduos de ambos os sexos, maiores de 18 anos, com o diagnóstico confirmado de HAS e/ou DM, que já possuíam registrado no prontuário pelo menos uma consulta médica e que aceitaram participar da pesquisa assinando o Termo de Consentimento Livre e Esclarecido (TCLE). Foram exclusas gestantes e aqueles que não foram encontrados em seus domicílios após três tentativas de contato, em horários e dias da semana diferentes.

A partir desses critérios, foram levantados 184 prontuários de usuários com HAS e/ou DM de acordo as informações nos registros da unidade de ESF. Destes, 06 não tinham diagnosticado nenhuma das doenças, 35 haviam mudado de endereço, 13 possuíam diagnóstico de óbito, 09 não foram localizados após três tentativas e 01 recusou-se a participar da pesquisa. Foi realizado um teste-piloto com 10 indivíduos com a finalidade de testar a adequação do questionário e dos procedimentos propostos. Estes usuários não participaram da amostra final da pesquisa, obtendo-se 110 participantes.

A coleta de dados aconteceu a partir de inquéritos domiciliares individualizados, através da aplicação de um questionário elaborado pelos pesquisadores, estruturado e organizado com as seguintes variáveis: dados sociodemográficos, clínicos, fatores de risco, dados antropométricos e morbidade auto referida.

As variáveis sociodemográficas analisadas foram sexo, faixa etária, cor auto referida, escolaridade (em anos de estudo), situação conjugal, moradores por domicílio, atividade de trabalho e classe econômica. Esta última foi classificada segundo o Critério de Classificação Econômica do Brasil da Associação Brasileira de Empresas de Pesquisa (2016), utilizando informações sobre a posse de determinados itens de conforto, presença de empregados domésticos e escolaridade do chefe da família; enquadrando as pessoas nas classes de A a $E$, a partir dos cortes alcançados.

As variáveis clínicas foram: o diagnóstico e rastreamento da doença, uso de medicação, última consulta, acompanhamento da doença e complicação da HAS e DM auto referidas. Além disso, foi verificada a pressão arterial diastólica (PAD) e sistólica (PAS) dos participantes conforme as recomendações da $7^{\circ}$ Diretriz Brasileira de Hipertensão (SOCIEDADE BRASILEIRA DE CARDIOLOGIA, 2016) para maiores de 18 anos: normotenso (PAD $\leq 80 \mathrm{mmHg} /$ PAS $\leq 120 \mathrm{mmHg}$ ); hipertensos acima (PAD: $\geq 81 \mathrm{mmHg} / \mathrm{PAS} \geq 121 \mathrm{mmHg}$ ).

Quanto aos fatores de risco analisou-se a hereditariedade para HAS e DM, tabagismo (considerado fumante regular consumo de 100 cigarros na vida e continua fumando) (BRASIL, 2015), sedentarismo, etilismo (considerado mais de 2 doses/dia) (WHO, 2010), ingestão de sal (5g/dia) (WHO, 2012) e açúcar (25g/dia) (WHO, 2015) e excesso de peso (IMC acima de 25) (BRASIL, 2011b). Em relação a morbidade auto referida considerou-se artrite/artrose/reumatismo, asma, sintomas de depressão e ansiedade, bronquite e problemas de audição. 
As medidas antropométricas foram coletadas segundo as recomendações do Ministério da Saúde (BRASIL, 2011b) e corresponderam ao peso registrado em quilograma $(\mathrm{Kg})$ e a estatura em centímetros $(\mathrm{cm})$. A mensuração do peso foi realizada com o indivíduo imóvel, sem sapato, com roupa leve e com os pés localizados no centro da balança. Utilizou-se uma balança digital portátil, com capacidade até $130 \mathrm{Kg}$ e precisão de 0,1 Kg. Já a mensuração da estatura foi realizada com o indivíduo em pé, com calcanhares, panturrilhas, região glútea, ombros e cabeça encostados em parede lisa e sem rodapés, com uma trena fixada, possuindo precisão de $0,5 \mathrm{~cm}$. A medida foi registrada com o indivíduo em posição anatômica.

Para avaliação do Índice de Massa Corporal (IMC), foi utilizada a seguinte classificação: IMC $\leq 18,5$ (baixo peso), IMC=18,5 a 24,9 (eutrófico), IMC $\geq 25$ a 29,9 (sobrepeso), IMC $\geq 30$ (obeso). A relação cintura / quadril (RCQ) e a circunferência abdominal (CA) foram avaliadas conforme os seguintes parâmetros: RCQ 0,85 kg/m2 para as mulheres e 0,95 kg/m2 para os homens. Para a CA, foi mensurado do ponto médio entre a borda inferior da última costela e a crista ilíaca lateral, sendo que os valores de normalidade adotados foram 80 $\mathrm{cm}$ para as mulheres e $94 \mathrm{~cm}$ para os homens (BRASIL, 2011b).

Os dados foram duplamente digitados no Microsoft Office Excel® 2007 e a verificação da consistência dos dados foi realizada utilizando-se o programa Epi Info®, versão 3.5.4. A significância estatística das diferenças entre as proporções foram testadas com o teste de qui-quadrado ou exato de Fisher, conforme mais apropriado. Para análise dos dados foram realizadas análises descritivas e razão de prevalência. Para associação entre os fatores de risco e morbidades auto referidas e a ocorrência de HAS e/ou DM foi utilizada razão de prevalência e intervalos de 95\% de confiança (IC95\%).

Esta pesquisa faz parte do projeto matricial intitulado "Consumo de Medicamentos por Usuários do SUS", que foi analisado e aprovado pelo Comitê de Ética em Pesquisa (CEP) do Hospital Universitário Júlio Muller, com CAAE 45225315.0.0000.5541. Portanto, foram respeitados todos os aspectos éticos e legais da pesquisa com seres humanos atendendo a Resolução 466/2012 (BRASIL, 2012c).

\section{RESULTADOS}

Participaram desta pesquisa 110 indivíduos, sendo $71,82 \%(n=79)$ hipertensos, $9,09 \%(n=10)$ diabéticos e $19,09 \%(n=21)$ hipertensos e diabéticos. A maioria é do sexo feminino $(58,18 \% ; n=64)$, faixa etária de 40 a 59 anos $(45,46 \%$; $n=$ $50)$, cor auto referida parda $(57,27 \% ; n=63)$, possuíam de 0 a 8 anos de estudo $(75,45 \% ; n=83)$, residiam com companheiro $(67,27 \% ; n=74)$ e com até 03 moradores por domicílio $(69,10 \%$; $n=76)$, não possuíam trabalho $(59,09 \%$; $n=$ 65 ) e pertenciam as classes sociais C e D $(99,10 \% ; n=109)$, conforme Tabela 1. 
Tabela 1 - Prevalência de hipertensão, diabetes e diabetes e hipertensão, segundo variáveis sociodemográficas de uma Estratégia Saúde da Família (ESF) de Rondonópolis-MT, 2017.

\begin{tabular}{|c|c|c|c|c|c|c|c|c|}
\hline \multirow[b]{2}{*}{ VARIAVEIS } & \multicolumn{2}{|c|}{ HAS } & \multicolumn{2}{|c|}{$\mathrm{DM}$} & \multicolumn{2}{|c|}{ HASIDM } & \multicolumn{2}{|c|}{ TOTAL HAS/DM } \\
\hline & $\mathrm{n}=79$ & $\%$ & $n=10$ & $\%$ & $n=21$ & $\%$ & $n=110$ & $\%$ \\
\hline Sexo & & & & & & & & \\
\hline $\begin{array}{l}\text { Masculino } \\
\text { Feminino }\end{array}$ & $\begin{array}{l}34 \\
45\end{array}$ & $\begin{array}{l}43,04 \\
56,96\end{array}$ & $\begin{array}{l}4 \\
6\end{array}$ & $\begin{array}{l}40 \\
60\end{array}$ & $\begin{array}{c}8 \\
13\end{array}$ & $\begin{array}{l}38,09 \\
61,91\end{array}$ & $\begin{array}{l}46 \\
64\end{array}$ & $\begin{array}{l}41,82 \\
58,18\end{array}$ \\
\hline $\begin{array}{l}\text { Faixa etária } \\
20-39 \\
40-59 \\
\text { Mais de } 80\end{array}$ & $\begin{array}{l}18 \\
38 \\
23\end{array}$ & $\begin{array}{l}22,79 \\
48,10 \\
29,11\end{array}$ & $\begin{array}{l}1 \\
4 \\
5\end{array}$ & $\begin{array}{l}10 \\
40 \\
50\end{array}$ & $\begin{array}{c}8 \\
13\end{array}$ & $\begin{array}{l}38,10 \\
61,90\end{array}$ & $\begin{array}{l}18 \\
50 \\
41\end{array}$ & $\begin{array}{l}17,27 \\
45,46 \\
37,27\end{array}$ \\
\hline $\begin{array}{l}\text { Raça } \\
\text { Preta } \\
\text { Branca } \\
\text { Amarela } \\
\text { Indígena } \\
\text { Parda }\end{array}$ & $\begin{array}{c}22 \\
13 \\
\\
1 \\
43\end{array}$ & $\begin{array}{c}27,85 \\
16,45 \\
\\
1,27 \\
54,43\end{array}$ & 7 & 70 & $\begin{array}{c}2 \\
4 \\
1 \\
1 \\
13\end{array}$ & $\begin{array}{c}9,52 \\
19,05 \\
4,78 \\
4,76 \\
61,91\end{array}$ & $\begin{array}{c}27 \\
17 \\
1 \\
2 \\
63\end{array}$ & $\begin{array}{c}24,55 \\
15,45 \\
0,91 \\
1,82 \\
57,27\end{array}$ \\
\hline $\begin{array}{l}\text { Escolaridade } \\
0-8 \text { anos } \\
\text { Mais de } 9 \text { anos }\end{array}$ & $\begin{array}{l}58 \\
21\end{array}$ & $\begin{array}{l}73,42 \\
26,58\end{array}$ & $\begin{array}{l}6 \\
4\end{array}$ & $\begin{array}{l}60 \\
40\end{array}$ & $\begin{array}{c}19 \\
2\end{array}$ & $\begin{array}{c}90,5 \\
9,5\end{array}$ & $\begin{array}{l}83 \\
27\end{array}$ & $\begin{array}{l}75,45 \\
24,55\end{array}$ \\
\hline $\begin{array}{l}\text { Situaçäo conjugal } \\
\text { Com companheiro (a) } \\
\text { Sem companheiro (a) }\end{array}$ & $\begin{array}{l}55 \\
24\end{array}$ & $\begin{array}{l}69,62 \\
30,38\end{array}$ & $\begin{array}{l}7 \\
3\end{array}$ & $\begin{array}{l}70 \\
30\end{array}$ & $\begin{array}{c}12 \\
9\end{array}$ & $\begin{array}{l}57,14 \\
42,86\end{array}$ & $\begin{array}{l}74 \\
36\end{array}$ & $\begin{array}{l}87,27 \\
32,73\end{array}$ \\
\hline $\begin{array}{l}\text { Moradores por domicilio } \\
\text { Até } 3 \\
24\end{array}$ & $\begin{array}{l}54 \\
25\end{array}$ & $\begin{array}{l}68,35 \\
31,65\end{array}$ & $\begin{array}{l}6 \\
4\end{array}$ & $\begin{array}{l}60 \\
40\end{array}$ & $\begin{array}{c}16 \\
5\end{array}$ & $\begin{array}{r}76,19 \\
23,81\end{array}$ & $\begin{array}{l}76 \\
34\end{array}$ & $\begin{array}{l}69,10 \\
30,90\end{array}$ \\
\hline $\begin{array}{l}\text { Classe Económica } \\
\text { Ae B } \\
\text { C e D }\end{array}$ & $\begin{array}{c}1 \\
78\end{array}$ & $\begin{array}{c}1,27 \\
98,73\end{array}$ & 10 & 100 & 21 & 100 & $\begin{array}{c}1 \\
109\end{array}$ & $\begin{array}{c}0,90 \\
99,10\end{array}$ \\
\hline $\begin{array}{l}\text { Trabalho } \\
\text { Sim } \\
\text { Não }\end{array}$ & $\begin{array}{l}35 \\
44\end{array}$ & $\begin{array}{l}44,30 \\
55,70\end{array}$ & $\begin{array}{l}6 \\
4\end{array}$ & $\begin{array}{l}60 \\
40\end{array}$ & $\begin{array}{c}4 \\
17\end{array}$ & $\begin{array}{l}19,05 \\
80,95\end{array}$ & $\begin{array}{l}45 \\
65\end{array}$ & $\begin{array}{l}40,81 \\
59,09\end{array}$ \\
\hline
\end{tabular}

No que tange as variáveis clínicas, a maioria dos participantes tem diagnosticado a doença há mais de 02 anos $(89,09 \% ; n=98)$, realizou o rastreamento da doença devido ao aparecimento de sinais e sintomas $(59,10 \% ; n=65)$, utilizam medicamentos $(95,45 \% ; n=105)$ e realizou a última consulta nos últimos 3 meses (60\%; $n=66)$. Verificou-se que $95,45 \% \quad(n=105)$ realizavam acompanhamento da doença, sendo a ESF o principal local frequentado pelos participantes $(93,33 \% ; n=98)$.

Dentre as complicações auto referidas houve prevalência de angina $(23,63 \%$; $n=$ 26) e problemas de visão $(16,36 \% ; n=18)$. Entretanto, $57,27 \%(n=63)$ não tiveram complicação. Após a verificação da pressão arterial, a maioria $(66,36 \%$; $n=73$ ) encontrava-se com a PA alterada.

Quanto às medidas antropométricas, a maioria dos participantes foi classificada com obesidade $(41,82 \%, n=46)$, prevalência de $R C Q$ de risco entre mulheres $(52,73 \% ; n=58)$ e homens $(38,18 \% ; n=42)$ e CA de risco entre as mulheres $(54,54 \% ; n=60)$ e homens $(30,91 \% ; n=34)$ (Tabela 2) 
Tabela 2 - Prevalência de hipertensão, diabetes e diabetes e hipertensão, segundo variáveis clínicas e medidas antropométricas em uma Estratégia Saúde da Família (ESF) de Rondonópolis-MT, 2017.

\begin{tabular}{|c|c|c|c|c|c|c|c|c|}
\hline \multirow[b]{2}{*}{ VARIAVEIS } & \multicolumn{2}{|c|}{ HAS } & \multicolumn{2}{|c|}{ DM } & \multicolumn{2}{|c|}{ HESIDIM } & \multicolumn{2}{|c|}{ TOTAL HASDOM } \\
\hline & $n=79$ & $\%$ & $n=10$ & $\%$ & $n=21$ & $\%$ & $n=110$ & $n=79$ \\
\hline \multicolumn{9}{|l|}{$\begin{array}{l}\text { Dlagnostico da } \\
\text { doença }\end{array}$} \\
\hline $\begin{array}{l}\text { Ha } 01 \text { ano } \\
\text { Ha }>-2 \text { anos }\end{array}$ & $\begin{array}{c}8 \\
71\end{array}$ & $\begin{array}{l}10,13 \\
89,87\end{array}$ & $\begin{array}{l}2 \\
8\end{array}$ & $\begin{array}{l}20 \\
80\end{array}$ & $\begin{array}{c}2 \\
19\end{array}$ & $\begin{array}{r}9,52 \\
90,48\end{array}$ & $\begin{array}{l}12 \\
98\end{array}$ & $\begin{array}{l}10,91 \\
89,09\end{array}$ \\
\hline \multicolumn{9}{|l|}{$\begin{array}{l}\text { Rastreamento da } \\
\text { doença }\end{array}$} \\
\hline $\begin{array}{l}\text { Sinals e sintomas } \\
\text { Exames de Rotina }\end{array}$ & $\begin{array}{l}51 \\
26\end{array}$ & $\begin{array}{l}64,56 \\
32,91\end{array}$ & $\begin{array}{l}5 \\
4\end{array}$ & $\begin{array}{l}50 \\
40\end{array}$ & $\begin{array}{c}9 \\
11\end{array}$ & $\begin{array}{l}42,86 \\
52,38\end{array}$ & $\begin{array}{l}65 \\
41\end{array}$ & $\begin{array}{l}59,10 \\
37,27\end{array}$ \\
\hline Outros & 2 & 2,63 & 1 & 10 & 1 & 4,76 & 4 & 3,63 \\
\hline \multicolumn{9}{|l|}{ Uso de medlcaçao } \\
\hline $\begin{array}{l}\text { Sim } \\
\text { Nabo }\end{array}$ & $\begin{array}{c}76 \\
3\end{array}$ & $\begin{array}{c}96,20 \\
3,80\end{array}$ & $\begin{array}{l}9 \\
1\end{array}$ & $\begin{array}{l}90 \\
10\end{array}$ & $\begin{array}{c}20 \\
1\end{array}$ & $\begin{array}{c}95,24 \\
4,76\end{array}$ & $\begin{array}{c}105 \\
5\end{array}$ & $\begin{array}{c}95,45 \\
4,55\end{array}$ \\
\hline \multicolumn{9}{|l|}{ ÓltIma Consulta } \\
\hline $\begin{array}{l}\text { Ate } 3 \text { meses } \\
4 \text { meses ou mals }\end{array}$ & $\begin{array}{l}50 \\
29\end{array}$ & $\begin{array}{l}63,30 \\
36,70\end{array}$ & $\begin{array}{l}7 \\
3\end{array}$ & $\begin{array}{l}70 \\
30\end{array}$ & $\begin{array}{c}9 \\
12\end{array}$ & $\begin{array}{l}42,86 \\
57,14\end{array}$ & $\begin{array}{l}66 \\
44\end{array}$ & $\begin{array}{l}60 \\
40\end{array}$ \\
\hline \multicolumn{9}{|c|}{$\begin{array}{l}\text { Acompanhamento da } \\
\text { doença }\end{array}$} \\
\hline Sim & $\begin{array}{c}74 \\
5\end{array}$ & $\begin{array}{c}93,68 \\
6,32\end{array}$ & 10 & 100 & 21 & 100 & $\begin{array}{c}105 \\
5\end{array}$ & $\begin{array}{r}95,45 \\
4,54\end{array}$ \\
\hline $\begin{array}{l}\text { ESF } \\
\text { Cardo SUS }\end{array}$ & $\begin{array}{c}70 \\
5\end{array}$ & $\begin{array}{c}94,59 \\
6,76\end{array}$ & 8 & 80 & 20 & 95,24 & $\begin{array}{c}98 \\
5\end{array}$ & $\begin{array}{c}93,33 \\
4,76\end{array}$ \\
\hline Cardlo Plano & 6 & 8,10 & & & & & 6 & 5,71 \\
\hline Endocrino Sus & & & 2 & 20 & 1 & 4.76 & 3 & 2,86 \\
\hline Outro & 1 & 1,35 & & & 1 & 4,76 & 2 & 1,90 \\
\hline \multicolumn{9}{|l|}{ Complicaçäo } \\
\hline Sim & 33 & 41,77 & 10 & 100 & 4 & 19,05 & 47 & 42.72 \\
\hline $\begin{array}{l}\text { Nalo } \\
\text { AVE }\end{array}$ & 46 & $\begin{array}{c}58,23 \\
6,06\end{array}$ & & & 18 & 85,71 & $\begin{array}{c}63 \\
2\end{array}$ & $\begin{array}{l}57,27 \\
1.81\end{array}$ \\
\hline LAM & 8 & 24,24 & & & 3 & 14,28 & 11 & $\begin{array}{l}1,01 \\
10\end{array}$ \\
\hline $\begin{array}{l}\text { Pe dlabetico } \\
\text { Amputaçăo }\end{array}$ & & & & & & & & \\
\hline Angina & 21 & 63,63 & 5 & 50 & & & 26 & 23,63 \\
\hline $\begin{array}{l}\text { Problemas de visâo } \\
\text { Outras }\end{array}$ & 4 & 12,12 & $\begin{array}{l}9 \\
1\end{array}$ & $\begin{array}{l}90 \\
10\end{array}$ & 5 & 23,80 & $\begin{array}{c}18 \\
1\end{array}$ & $\begin{array}{c}16,36 \\
0,90\end{array}$ \\
\hline \multicolumn{9}{|l|}{ PA } \\
\hline Normotenso & 23 & 29,12 & 7 & 70 & 7 & 33,34 & 37 & 33,64 \\
\hline Hipertenso & 56 & 70,88 & 3 & 30 & 14 & 66,66 & 73 & 66,36 \\
\hline \multicolumn{9}{|l|}{ IMC } \\
\hline Balxo peso & 1 & 1,26 & & & 1 & 4,76 & 2 & 1,82 \\
\hline Eutrotco & 16 & 20,25 & 3 & 30 & 2 & 9.52 & 21 & 19.09 \\
\hline Sobrepeso & 29 & 36,71 & 6 & 60 & 6 & 28,57 & 41 & 37,27 \\
\hline Obesidade & 33 & 41,77 & 1 & 10 & 12 & 57,15 & 46 & 41,82 \\
\hline
\end{tabular}




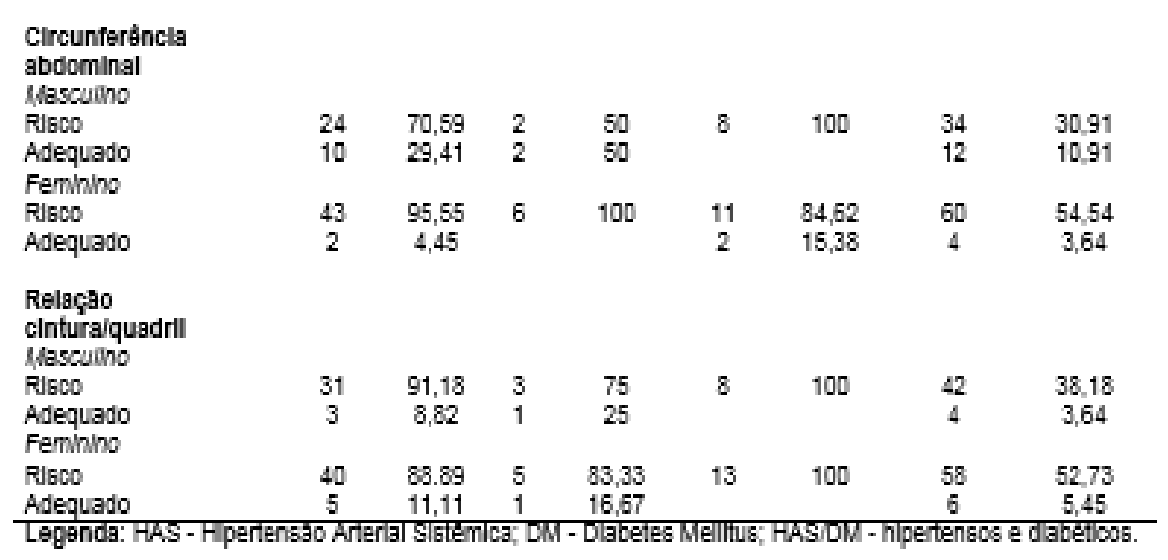

Os hipertensos apresentaram menor prevalência (64\%; $n=64)$ de morbidade auto referida, em relação aos diabéticos $(74,19 \% ; n=23)$, hipertensos $(65,62 \% ; n=42)$ e diabéticos $(39,13 \% ; n=9)$ referiram sintomas de ansiedade. Porém, apenas os indivíduos hipertensos apresentaram associação estatística com artrite/artrose/reumatismo $(p=0,017)$ e ansiedade $(p=0,045)$. Em relação as morbidades auto referidas em diabéticos, não houve associação estatística (Tabela 3). 
Tabela 3 - Prevalência e razão de prevalência dos fatores de risco e morbidades auto referidas em hipertensos e diabéticos uma Estratégia Saúde da Família (ESF) de Rondonópolis-MT, 2017.

\begin{tabular}{|c|c|c|c|c|c|c|c|c|}
\hline \multirow[b]{2}{*}{ VARIAVEL } & \multicolumn{4}{|c|}{ TOTAL HAS } & \multicolumn{4}{|c|}{ TOTAL DM } \\
\hline & $n=100$ & $\%$ & p & $\begin{array}{c}\mathrm{RP} \\
\text { (IC95\%) }\end{array}$ & $n=31$ & $\%$ & p & RP (IC95\%) \\
\hline \multicolumn{9}{|l|}{ Fatores de Risco } \\
\hline Sim & 93 & 93 & \multirow[t]{2}{*}{0,034} & \multirow[t]{2}{*}{$\begin{array}{c}0,90 \\
(0,84-0,96)\end{array}$} & 30 & 96,77 & \multirow[t]{2}{*}{0,168} & \multirow[t]{2}{*}{$\begin{array}{c}2,03 \\
(0,32-12,83)\end{array}$} \\
\hline Não & 7 & 7 & & & 1 & 3,23 & & \\
\hline Hereditariedade HAS & 60 & 64,52 & 0,078 & \multirow{3}{*}{$\begin{array}{c}1,03 \\
(0,81-1,17) \\
0,85 \\
(0,72-0,99) \\
0,93 \\
(0,78-1,12)\end{array}$} & 1 & 56,68 & 0,124 & \multirow{3}{*}{$\begin{array}{c}0,84 \\
(0,46-1,52) \\
2,82 \\
(1,44-4,75) \\
0,59 \\
(0,23-1,51)\end{array}$} \\
\hline Hereditariedade DM & 31 & 33,33 & 4,512 & & 18 & 60 & 0,860 & \\
\hline Tabagismo & 19 & 20,43 & 0,171 & & 4 & 13,33 & 0,185 & \\
\hline Sedentarismo & 51 & 54,83 & 2,018 & \multirow{5}{*}{$\begin{array}{c}0,89 \\
(0,80-1,00) \\
0,96 \\
(0,84-1,10) \\
1,06 \\
(0,85-1,19) \\
1,04 \\
(0,90-1,19) \\
0,95 \\
(0,84-1,07)\end{array}$} & 19 & 83,33 & 0,833 & \multirow{5}{*}{$\begin{array}{c}1,38 \\
(0,73-2,53) \\
0,84 \\
(0,43-1,63) \\
0,40 \\
(0,13-1,21) \\
0,37 \\
(0,09-1,43) \\
1,18 \\
(0,65-2,16)\end{array}$} \\
\hline Ingestăo sal & 32 & 34,40 & 0,025 & & 8 & 30 & 0,085 & \\
\hline Ingestão Açúcar & 22 & 23,85 & 0,232 & & 3 & 10 & 0,055 & \\
\hline Ingere Alcool & 16 & 17,20 & 0,0017 & & 2 & 6,66 & 0,084 & \\
\hline Excesso de Peso & 46 & 48,46 & 0,283 & & 16 & 53,33 & 0,128 & \\
\hline \multicolumn{9}{|l|}{ Morbidade auto referida } \\
\hline & 64 & 81,01 & \multirow[t]{2}{*}{0,001} & \multirow[t]{2}{*}{$\begin{array}{c}0,97 \\
(0,86-1,09)\end{array}$} & 23 & 74,19 & \multirow[t]{2}{*}{1,217} & \multirow[t]{2}{*}{$\begin{array}{c}1,57 \\
(0,78-3,18)\end{array}$} \\
\hline Nâo & 36 & 32,81 & & & 8 & 25,81 & & \\
\hline Artrite/artrose/reumatismo & 14 & 21,87 & 0,017 & \multirow{2}{*}{$\begin{array}{c}1,03 \\
(0,88-1,19) \\
1,10 \\
(1,03-1,17)\end{array}$} & 6 & 26,08 & 0,817 & \multirow[t]{2}{*}{$\begin{array}{c}1,52 \\
(0,75-3,07)\end{array}$} \\
\hline Asma & 2 & 3,125 & 0,825 & & & & & \\
\hline Sintomas de Depressão & 9 & 14,06 & 0,148 & \multirow{4}{*}{$\begin{array}{c}1,10 \\
(1,04-1,18) \\
1,00 \\
(0,89-1,13) \\
0,87 \\
(0,56-1,36) \\
1,10 \\
(1,03-1,17)\end{array}$} & 1 & 4,34 & 0,218 & \multirow{4}{*}{$\begin{array}{c}0,37 \\
(0,05-2,43) \\
0,58 \\
(0,28-1,11) \\
1,44 \\
(0,47-4,42) \\
1,80 \\
(0,43-7,43)\end{array}$} \\
\hline Sintomas de Ansiedade & 42 & 65,82 & 0,045 & & 9 & 39,13 & 0,067 & \\
\hline Bronquite & 4 & 6,25 & 0,384 & & 2 & 8,69 & 0,435 & \\
\hline Problemas de audição & 2 & 3,12 & 0,825 & & 1 & 4,34 & 0,486 & \\
\hline
\end{tabular}

A prevalência de fatores de risco em hipertensos foi de 93\% (IC95\%: 0,84-0,96), com destaque a hereditariedade para HAS $(64,52 \% ; n=60)$ e o sedentarismo $(54,83 \% ; n=51)$.

Enquanto em diabéticos foi de 96,77\% (IC95\%: 0,32-12,83), com prevalência do sedentarismo $(63,33 \% ; n=19)$; hereditariedade para HAS $(56,66 \% ; n=17)$ e 0 excesso de peso (53,33\%; $n=16)$. Em relação a associação entre os fatores de risco dos hipertensos, foi encontrada associação estatística significativa com a ingestão de sal $(p=0,025)$ e ingestão de álcool $(p=0,001)$. Quanto aos fatores de risco dos diabéticos houve relevância apenas em relação à ingestão de açúcar $(p=$ 0,055). 


\section{DISCUSSÃO}

Os resultados do presente estudo evidenciaram a prevalência de HAS e/ou DM em mulheres (58,18\%); a prevalência do sexo feminino também foi demonstrada em estudos semelhantes (GOIS et al, 2016; WHO, 2013ª; DICOW, 2015). Este fato pode estar relacionado às alterações hormonais decorrente do climatério e menopausa, fragilizando a mulher no contexto cardiovascular (GORGUI et al, 2014). Em 2014, dados do IBGE demonstraram que no Brasil houve maior prevalência de DM em mulheres (7,0\%), enquanto nos homens foi de 5,4\%. Por outro lado, a maior procura pelos serviços de saúde pelas mulheres pode representar maior oportunidade de diagnóstico médico de HAS e do DM (ASSIS; JESUS, 2012).

A faixa etária predominante deste estudo foi de 40 a 59 anos (45,46\%); em estudo similar de Sales et al. (2013), a maioria dos portadores de HAS também encontravam-se na faixa etária acima de 40 anos de idade. Apesar de não predominante, os idosos $(37,27 \% ; n=41)$ também possuem altos índices de diagnósticos destas DCNT neste local de estudo. A $7^{\text {a }}$ Diretriz Brasileira de Hipertensão (SOCIEDADE BRASILEIRA DE CARDIOLOGIA, 2016) destaca relação direta e linear da faixa etária maior que 60 anos com a prevalência da HAS. Outros estudos também demonstraram elevadas taxas de prevalência da DM na população idosa, o que sugere que o processo de envelhecimento possui associação positiva com o aumento destas doenças, como o enrijecimento da artéria aorta, maiores resistências vascular periférica e à insulina (SOCIEDADE BRASILEIRA DE DIABETES, 2016; GOMIDE et al, 2013; DICOW, 2015; MIKAEL, 2017).

Em relação à cor da pele auto referida, Lotufo \& Bensenor (2013) apontam que a morbimortalidade em decorrência da HAS e de outras doenças crônicas é maior entre pessoas de raça/cor da pele preta, corroborando com o estudo ELSA-Brasil (CHOR et al, 2015) que mostrou prevalências de 49,3\% em negros, $30,3 \%$ em brancos, $38,2 \%$ em pardos. Porém neste estudo houve predomínio de pessoas com cor auto referida parda $(57,27 \%)$. Tal dado condiz com estudo desenvolvido em Macapá (AP) (CHAGAS; ALMEIDA, 2016) que também constatou predomínio da HAS em pardos $61,2 \%$. Em relação ao DM a Sociedade Brasileira de Diabetes (SOCIEDADE BRASILEIRA DE DIABETES, 2016) não evidencia relação entre a cor da pele e a doença, portanto, o que pode justificar a presença maior de pardos no estudo é que esta é a segunda raça predominante no Brasil (46,7\% total da população) (IBGE, 2016).

No que se refere à escolaridade, este estudo verificou que $75,45 \%$ dos participantes possuíam de 0 a 8 anos de estudo. Similarmente, Pereira et al. (2014), constataram um número expressivo de baixa escolaridade $(79,4 \%)$ entre hipertensos em Santa Maria (RS). Da mesma maneira em relação ao DM, outros estudos sugerem maior taxa de diagnóstico em indivíduos com baixa escolaridade (BRASIL, 2017; SILVA et al, 2015). Estudo realizado por Brischiliari et al. (2014) identificou que o desenvolvimento das DCNT tende a aumentar em indivíduos com baixa escolaridade, visto que pode interferir na adesão ao tratamento, considerando a necessidade do indivíduo em seguir o plano terapêutico em relação às medicações e alimentação.

Quanto à situação conjugal, este estudo verificou que $67,27 \%$ dos participantes viviam com companheiro e $69,10 \%$ com até 03 moradores por domicílio, 
similarmente aos estudos de Pereira et al. (2014) e Ferreira \& Ferreira (2009), em que $59 \%$ dos hipertensos e cerca de $90 \%$ diabéticos, respectivamente, também conviviam com companheiro ou outras pessoas. Outras pesquisas demonstraram que há estreita relação do sucesso do plano terapêutico com o apoio familiar (MAGMABOSCO et al, 2015; SILVA et al, 2015).

As classes sociais C e D predominaram (99,1\%) neste estudo. Outras publicações sugerem a desigualdade social na presença de condições crônicas, especificamente no que se refere à HAS e ao DM, considerando que o indivíduo em situação econômica mais desfavorável estaria mais exposto a uma série de fatores de risco (DICOW, 2015; WHO, 2013a).

Dos participantes, $89,09 \%$ têm diagnóstico da doença há mais de 02 anos e $59,10 \%$ realizaram o rastreamento da doença devido ao aparecimento de sinais e sintomas. De acordo com Magnabosco et al. (2015) indivíduos com maior tempo de diagnóstico da doença apresentam maior adesão ao tratamento. Semelhantemente, Motter et al. (2015) apontam que o maior tempo de diagnóstico, pressupõe maior utilização dos serviços de saúde, proporcionando também maior exposição do portador às orientações necessárias para o controle da doença. Entretanto, a procura do rastreamento apenas após o aparecimento dos sinais e sintomas, representa falha no cumprimento dos princípios e diretrizes da atenção primária a saúde (BRASIL, 2014).

Quanto ao acompanhamento da doença $95,45 \%$ realizavam e referiram última consulta nos últimos 3 meses, sendo a ESF (93,33\%) o principal local citado. Em Serra Talhada (PE), a frequência de acompanhamento na ESF foi observada em $66,15 \%$ dos indivíduos. Nesse sentido, o vínculo criado com a ESF pode ser considerado um fator que facilita o adequado manejo da doença (LIMA et al, 2012).

Um achado importante deste estudo é que, apesar destas doenças terem complicações incapacitantes, $57,27 \%$ não tiveram nenhuma complicação. Entretanto, verificou-se durante aferição de PA nas visitas domiciliares que $66,36 \%$ dos participantes encontravam-se hipertensos. Embora o risco seja mais alto naqueles indivíduos com níveis mais elevados de PA, esta categoria geralmente passa despercebida pelos hipertensos e acaba sendo responsável por grande proporção de morbidade e incapacidades atribuíveis a doença (SOCIEDADE BRASILEIRA DE CARDIOLOGIA, 2016).

Cerca de 93\% (RP: 0,90 IC95\%: 0,84-0,96) dos hipertensos apresentaram fatores de risco, enquanto nos diabéticos foi 96,77\% (RP: 2,03 IC95\%: 0,3212,83). Entre os fatores de risco prevalentes neste estudo está a obesidade e ingestão de sal, álcool e açúcar. Malta et al. (2017) relacionam o excesso de peso/obesidade como gradiente positivo para prevalência da HAS, enquanto a 7a Diretriz Brasileira de Hipertensão (SOCIEDADE BRASILEIRA DE CARDIOLOGIA, 2016) descreve que perder peso constitui o método mais efetivo para redução da pressão arterial em obesos, além de contribuir para diminuição das doses de medicamentos anti-hipertensivos.

Assim como a ingestão inadequada e excessiva de sal, um dos principais fatores de risco para HAS associa-se a eventos cardiovasculares e renais. Como também a ingestão do álcool associou-se a alterações na PA e a morbimortalidade cardiovascular (SPOSITO et al, 2007; HE; MACGREGOR, 2010; SOUZA; PÓVOA, 2014).

$\mathrm{Na}$ prevalência de morbidade auto referida em hipertensos 64\% (RP: 0,97 IC95\%: 0,86-1,07), destacou-se a artrite/artrose/reumatismo $(p=0,017)$ e sintomas ansiedade $(p=0,045)$. Estudo de Pereira et al. (2012) aponta a HAS como um dos principais fatores de risco modificáveis para doença cardiovascular 
em pacientes com artrite reumatoide. Enquanto, Edmondson et al. (2015), constataram que os aumentos momentâneos da ansiedade estão associados ao aumento da pressão arterial.

\section{CONSIDERAÇÕES FINAIS}

O presente artigo mostrou que, comparativamente aos homens, as mulheres representam maior prevalência de HAS e/ou DM, destacando baixos níveis de escolaridade e classe social. Quanto aos fatores de risco, demonstrou associação para HAS entre a ingestão de sal e álcool e para DM de açúcar. A finalidade desta pesquisa evidencia a importância de conhecer o perfil epidemiológico de hipertensos e/ou diabéticos de um território, devido à possibilidade de identificar os fatores de risco para o agravo destas doenças, considerando que estas atuam silenciosamente, sendo as consequências de suas complicações, muitas vezes, irreversíveis. Portanto, a redução destes fatores modificáveis é fundamental para o controle, prevenção de agravos, e consequentemente redução da morbimortalidade associada à HAS/DM.

Uma limitação do estudo foi o tamanho reduzido da amostra, que se deve ao fato de ter sido estabelecido para somente uma unidade de ESF, além de que o levantamento de registro no prontuário foi dificultado pelo fato da não realização de acompanhamento eficaz destes usuários. Considerando a relevância desta temática, sugere-se que novas pesquisas sejam realizadas, a fim de analisar os multifatores e determinar outros possíveis riscos expostos para estas doenças.

\section{REFERÊNCIAS}

ASSIS, M. M. A.; JESUS, W. L. A. Acesso aos serviços de saúde: abordagens, conceitos, políticas e modelo de análise. Ciência e Saúde Coletiva, v, 17, n. 11, p. 2865-75, 2012. Disponível em:

http://www.scielo.br/pdf/csc/v17n11/v17n11a02.pdf.

ASSOCIAÇÃO BRASILEIRA DE EMPRESAS DE PESQUISA. Critério de classificação econômica Brasil: Critério Brasil 2015 e atualização da distribuição de classes para 2016. ABEP, 2016.

BRASIL. Ministério da Saúde. Coordenação Nacional de Hipertensão e Diabetes. Diabetes Mellitus. Brasília: Ministério da Saúde, 2011a.

BRASIL. Ministério da Saúde. Diretrizes para o cuidado das pessoas com doenças crônicas nas redes de atenção à saúde e nas linhas de cuidado prioritárias. Brasília: Ministério da Saúde, 2013. 30p. Disponível em: http://bvsms.saude.gov.br/bvs/publicacoes/diretrizes\%20_cuidado_pessoas\%2 $0 \_$doencas_cronicas.pdf.

BRASIL. Ministério da Saúde. Estratégias para o cuidado da pessoa com doença crônica: o cuidado da pessoa tabagista. Brasília, Ministério da Saúde, 2015. 154p. Disponível em:

http://189.28.128.100/dab/docs/portaldab/publicacoes/caderno_40.pdf.

BRASIL. Ministério da Saúde. Norma Técnica do Sistema de Vigilância

Alimentar e Nutricional - SISVAN. Brasília: Ministério da Saúde, 2011b. 76p. Disponível em: 
http://bvsms.saude.gov.br/bvs/publicacoes/orientacoes_coleta_analise_dados antropometricos.pdf.

BRASIL. Ministério da Saúde. Portaria no 483, de 1ํ de Abril de 2014.

Redefine a Rede de Atenção à Saúde das Pessoas com Doenças Crônicas no âmbito do Sistema Único de Saúde (SUS) e estabelece diretrizes para a organização das suas linhas de cuidado. Conselho Nacional de Saúde, 2014.

BRASIL. Ministério da Saúde. Saúde Brasil 2011: uma análise da situação de saúde e a vigilância da saúde da mulher. Brasília: Ministério da Saúde, 2012a. 446p. Disponível em:

http://bvsms.saude.gov.br/bvs/publicacoes/saude_brasil_2011.pdf.

BRASIL. Ministério da Saúde. Vigitel Brasil 2011: vigilância de fatores de risco e proteção para doenças crônicas por inquérito telefônico. Brasília, Ministério da Saúde, 2012b. 136p. Disponível em:

http://bvsms.saude.gov.br/bvs/publicacoes/vigitel_brasil_2011.pdf.

BRASIL. Ministério da Saúde. Vigitel Brasil 2014: vigilância de fatores de risco e proteção para doenças crônicas por inquérito telefônico. Brasília, Ministério da Saúde, 2015. 152p. Disponível em:

http://bvsms.saude.gov.br/bvs/publicacoes/vigitel_brasil_2014.pdf.

BRASIL. Ministério da Saúde. Vigitel Brasil 2016: vigilância de fatores de risco e proteção para doenças crônicas por inquérito telefônico. Brasília: Ministério da Saúde, 2017. 162p. Disponível em:

http://portalarquivos.saude.gov.br/images/pdf/2017/junho/07/vigitel_2016_jun17 .pdf.

BRASIL. Portaria $n^{\circ}$ 466/2012 de outubro de 2012. Dispõe sobre diretrizes e normas regulamentadoras de pesquisa com seres humanos. Brasília (DF): Conselho Nacional de Saúde, 2012c. Publicada no Diário Oficial da União de 13 de junho de 2013; Seção 1: 59.

BRISCHILIARI, S. C. R.; AGNOLO, C. M. D.; GRAVENA, A. A. F.; LOPES, T. C. R.; CARVALHO, M. D. B.; PELLOSO, S. M. Doenças Crônicas não Transmissíveis e Associação com Fatores de Risco. Rev Bras Cardiologia, v. 27, n. 1, p. 531-428, 2014. Disponível em: http://www.rbconline.org.br/wpcontent/uploads/Art_52_RBC_27_1_Catia_Agnollo_Artigo_Original.pdf.

CHAGAS, J. A. S.; ALMEIDA, A. N. F. A. Caracterização epidemiológica de pacientes hipertensos usuários de uma unidade básica de saúde da Região Norte. Cad. de Educação, Saúde e Fisioterapia, v. 3, n. 6, p. 105-116, 2016.

CHOR, D.; PINHO, R. A. L.; SÁ, C. M.; DUNCAN, B. B.; ANDRADE, L. P.; ARAÚJO, N. A.; et al. Prevalence, awareness, treatment and influence of socioeconomic variables on control of high blood pressure: results of the ELSABrasil Study. Plos One, v.10, n. 6, p. 1-14, 2015. Disponível em: https://www.ncbi.nlm.nih.gov/pmc/articles/PMC4478044/.

CRUZ, M. F.; RAMIRES, V. V.; WENDT, A.; MIELKE, G. I.; MESA, J. M.; WEHRMEISTER, F. C. Simultaneidade de fatores de risco para doenças crônicas não transmissíveis entre idosos da zona urbana de pelotas, Rio grande do Sul, Brasil. Caderno de Saúde Pública, v. 33, n. 2, p. 1-11, 2017. 
Disponível em: http://www.scielo.br/pdf/csp/v33n2/1678-4464-csp-33-02e00021916.pdf.

DICOW, I. Perfil epidemiológico de pacientes portadores de Diabetes Mellitus tipo 2 residentes do município de Aguda, RS. Cinergis, v.16, n. 4, p. 261-66, 2015. Disponível em:

https://online.unisc.br/seer/index.php/cinergis/article/viewFile/6177/4744.

EDMONDSON, D.; ARNDT, J.; ALCÁNTARA, C.; CHAPLIN, W.; SCHWARTZ, J. E. Self-esteem and the acute effect of anxiety on ambulatory blood pressure. Psychosom Med., v. 77, n. 7, p. 833-841, 2015. Disponível em: https://www.ncbi.nlm.nih.gov/pmc/articles/PMC4565744/.

EVANGELISTA, A. S.; GUIMARÃES, M. C. S.; LEAL, M. B. Fluxos de Informação no SisHiperDia: um estudo de caso. Reciis, v. 7, n. 4, p. 1-14, 2013. Disponível em https://www.reciis.icict.fiocruz.br/index.php/reciis/article/view/570/pdf-570.

FERREIA, C. L. R. A.; FERREIRA, M. G. Características epidemiológicas de pacientes diabéticos da rede pública de saúde - análise a partir do sistema HiperDia. Arq Bras Endocrinol Metab, v. 53, n. 1, p. 80-86, 2009.

FREITAS, J. G. A.; NIELSON, S. E. O.; PORTO, C. C. Adesão ao tratamento farmacológico em idosos hipertensos: uma revisão integrativa da literatura. Rev Soc Clin Med, v. 13, n. 1, p. 75-84, 2017. Disponível em: http://files.bvs.br/upload/S/1679-1010/2015/v13n1/a4782.pdf.

GOIS, C. F. L.; SANTOS, J. F. S.; LIMA, A. C. R.; GONÇALVES, G. M.; SANTOS, F. L. L. S. M.; TEIXEIRA, J. R. M.; BARRETO, M. A. S. Perfil sociodemográfico e clínico de hipertensos atendidos por equipe da Saúde da Família. Rev Min Enferm, v. 20, n. 960, p. 1-6, 2016. Disponível em: http://pesquisa.bvsalud.org/portal/resource/pt/biblio-835269.

GOMIDE, N. A. C.; MOREIRA, O. C.; OLIVEIRA, R. A. R.; AMORIM, P. R. S. MARINS, J. C. B. Prevalência de glicemia sugestiva de Diabetes Mellitus e intolerância a glicemia de jejum em uma cidade do interior do Brasil. Arq.

Ciênc. Saúde Unipar, v. 17, n. 3, p. 147-152, 2013. Disponível em: http://www.revistas.unipar.br/index.php/saude/article/view/5063/2945.

GORGUI, J.; GORSHKOV, M.; KHAN, N.; DASKALOPOULOU, S. S. Hypertension as a risk factor for ischemic stroke in women. Can $\mathrm{J}$ Cardiol, v. 30, n. 7, p. 774-82, 2014. Disponível em: https://www.ncbi.nlm.nih.gov/pubmed/24970789.

HE, F. J.; MACGREGOR, G. A. Reducing population salt intake worldwide: from evidence to implementation. Prog Cardiovascular Diseases, v. 52, n. 5, p. 363-382, 2010. Disponível em:

https://www.ncbi.nlm.nih.gov/pubmed/20226955.

HOSEY, G. M; SAMO; M.; GREGG, E. W.; PADDEN, D.; BIBB, S. G.

Socioeconomic and demographic predictors of selected cardiovascular risk factors among living in Pohnpei, Federated States ok Micronesia. BMC Public Health, v. 14, n. 895, p. 1-14, 2014. Disponível em:

https://bmcpublichealth.biomedcentral.com/articles/10.1186/1471-2458-14-895. 
INSTITUTO BRASILEIRO DE GEOGRAFIA E ESTATÍSTICA (IBGE).

Pesquisa Nacional de Saúde 2013: percepção do estado de saúde, estilos de vida e doenças crônicas - Brasil, Grandes Regiões e Unidades da Federação. Rio de Janeiro: IBGE, 2014.

Instituto Brasileiro de Geografia e Estatística (IBGE). Síntese de Indicadores sociais - uma análise das condições de vida. Rio de Janeiro. IBGE. 2016.

INTERNATIONAL DIABETES FEDERATION. Diabetes atlas update 2015: Regional \& Country Facctsheets. 8 ed. Brussels: International Diabetes Federation, 2015. Disponível em:

<http://www.diabetesatlas.org/resources/2015-atlas.html>.

LIM, S. S.; VOS, T.; FLAXMAN, A. D.; DANAEI, G.; SHIBUYA, K.; ADAIRROHANI, H.; et al. A comparative risk assessment of burden of disease and injury attributable to 67 risk factors and risk factor clusters in 21 regions, 19902010: a systematic analysis for the Global Burden of Disease Study 2010. The Lancet, v. 380, n. 9859, p. 2224-60, 2012. Disponível em: https://www.ncbi.nlm.nih.gov/pubmed/23245609.

LIMA; A. S.; GAIA; E. S. M.; FERREIRA, M. A. A importância do Programa Hiperdia em uma Unidade de Saúde da Família do município de Serra Talhada-PE. Coletiva em Debates, v. 2, n. 1, p. 30-29, 2012. Disponível em: http://www.scielo.br/pdf/reben/v68n4/0034-7167-reben-68-04-0626.pdf.

LOBO, L. A. C.; CANUTO, R.; COSTA, J. S. D.; PATUSSI, M. P. Tendência temporal de hipertensão arterial sistêmica no Brasil. Caderno de Saúde Pública, v. 33, n. 6, p. 1-13, 2017. Disponível em: http://www.scielo.br/scielo.php?script=sci_arttext\&pid=S0102311 X2010000800001.

LOTUFO, P. A.; BENSENOR, I. J. M. Raça e mortalidade cerebrovascular no Brasil. Rev. Saúde Pública, v. 47, n. 6, p. 1201-1204, 2013. Disponível em: http://www.scielo.br/scielo.php?pid=S00349102013000601201\&script=sci_abst ract\&tlng $=$ pt.

MAGNABOSCO, P.; TERAOKA, E. C.; OLIVEIRA, E. M.; FELIPE, E. A.; FREITAS, D.; ALVES, L. M. M. Análise comparativa da não adesão ao tratamento medicamentoso da hipertensão arterial sistêmica em população urbana e rural. Rev. Latino Am Enfermagem, v. 23, n. 1, p. 20-27, 2015. Disponível em: http://www.scielo.br/pdf/rlae/v23n1/pt_0104-1169-rlae-23-0100020.pdf.

MALTA, D. C.; BERNAL, R. T. I.; ANDRADE, S. S. C. A; SILVA, M. M. A.; MELENDEZ, G. V. Prevalência e fatores associados com hipertensão arterial autorreferida em adultos brasileiros. Rev Saúde Pública, v. 51, n. 1, p. 1-11, 2017. Disponível em: http://www.scielo.br/scielo.php?pid=S003489102017000200313\&script=sci_arttext\&tlng=pt.

MIKAEL, L. R.; PAIVA, A. M. G.; GOMES, M. M.; SOUSA, A. L. L.; JARDIM, P. C. B.; VITORINO, P. V. O.; et al. Envelhecimento Vascular e Rigidez Arterialartigo de Revisão. Sociedade Brasileira de Cardiologia, v. 109, n. 2, p. 253258, 2017. Disponível em: http://www.scielo.br/pdf/abc/v109n3/pt_0066-782Xabc-20170091.pdf. 
MOTTER, F. R.; OLINTO, M. T. A.; PANIZ, V. M. V. Avaliação do conhecimento sobre níveis tensionais e cronicidade da hipertensão: estudo com usuários de uma Farmácia Básica no Sul do Brasil. Cad. Saúde Pública, v. 31, n. 2, p. 395-404, 2015. Disponível em: http://www.scielo.br/scielo.php?script=sci_arttext\&pid=S0102311 X2015000200395.

PEREIRA, C.; MARINHO, M.; CASSOLA, T.; SEERIG, A. P.; MUSSOI, T. D; BLÜMKE, A. C. Perfil Epidemiológico de Hipertensos e Diabéticos em uma Estratégia da Saúde da Família de Santa Maria/RS. Disciplinarum Scientia, v.15, n. 2, p. 195-203, 2014. Disponível em: https://www.periodicos.unifra.br/index.php/disciplinarumS/article/viewFile/1078/ 1022.

PEREIRA, I. A.; MOTA, M. H.; CRUZ, B. A.; BRENOL, C. V.; FRONZA, L. S. R.; BERTOLO, M. B.; et al. Consenso 2012 da Sociedade Brasileira de Reumatologia sobre o manejo de comorbidades em pacientes com artrite reumatoide. Rev Bras Reumatologia, v. 52, n. 4, p. 474-495, 2012. Disponível em: http://www.scielo.br/scielo.php?script=sci_arttext\&pid=S048250042012000400002.

PORTELA, R. S.; BARBOSA, A. M.; MARTINS, M. A.; PELAZZA, B. B.; LEITE, G. R.; PAULA, C. R. Prevalência de fatores de risco cardiovasculares e fatores associados em usuários de unidades de saúde. Rev Enferm UFPE, v. 10, n. 9 , p. 3232-3240, 2016. Disponível em:

https://periodicos.ufpe.br/revistas/revistaenfermagem/.

SALES, J. C. S.; BARROS, C. R. O.; MACHADO, I. M. L. N.; SANTOS, L. N. B.; CAMPELO, V.; COÊLHO, D. M. M.; et al. Perfil Epidemiológico dos Pacientes Hipertensos de um centro de Saúde, Teresina-PI. Teresina: Rev Multiprofissional de Saúde, v. 1, n. 1, p. 4-13, 2013. Disponível em: http://ojs.saomarcos.org.br/ojs/index.php/cientifica/article/view/14

SCALA, L. C.; MAGALHÃES, L. B.; MACHADO A. Epidemiologia da hipertensão arterial sistêmica. In: Moreira SM, Paola AV; Sociedade Brasileira de Cardiologia. Livro Texto da Sociedade Brasileira de Cardiologia. 2 ed. São Paulo: Manole, 2015. 780-85.

SILVA, A. L.; FONSECA, G. S. S.; ROSSI, V. E. C. Adesão ao tratamento medicamentoso entre pessoas com diabetes mellitus. Ciência et Praxis, v. 8, n. 16, p. 21-26, 2015. Disponível em: http://revista.uemg.br/index.php/praxys/article/view/2219.

SOCIEDADE BRASILEIRA DE CARDIOLOGIA. 7" Diretriz Brasileira de Hipertensão Arterial. Arq Bras Cardiologia, v. 107, n. 3, 2016. 83p. SOCIEDADE BRASILEIRA DE DIABETES. Diretrizes da Sociedade Brasileira de Diabetes. Ac Farmacêutica, 2016. 348p.

SOUZA, D.; PÓVOA, R. Álcool e Hipertensão arterial. Sociedade Portuguesa de Cardiologia, n.32, p. 33-39, 2014. Disponível em: http://ptdocz.com/doc/527982/\%C3\%A1lcool-e-hipertens\%C3\%A3o-arterial--sociedade-portuguesa-de-c.

SPOSITO, A. C.; CARAMELLI, B.; FONSECA, F. A. H.; BERTOLAMI, M. C. IV Diretriz brasileira sobre dislipidemias e prevenção da aterosclerose:

Departamento de Aterosclerose da Sociedade Brasileira de Cardiologia.

Arquivos Brasileiros de Cardiologia, v. 88, p. 2-19, 2007. Disponível em: 
http://www.scielo.br/scielo.php?script=sci_arttext\&pid=S0066-

782X2007000700002.

WORLD HEALTH ORGANIZ ATION. A global brief on hypertension: silent killer, global public health crisis. Geneva: WHO, 2013a.

WORLD HEALTH ORGANIZATION. Ingestão de açúcares por adultos e crianças. Geneva: WHO; 2015.

WORLD HEALTH ORGANIZATION. Self-Help strategies- for cutting down or stopping substance use. Geneva: WHO; 2010.

WORLD HEALTH ORGANIZATION. Sodium intake for adults and children. Geneva: WHO, 2012.

WORLD HEALTH ORGANIZATION. World health statistics. Geneva: World Health Organization, 2013b.

Recebido em: 22-10-2018

Aceito em: 17-03-2020 